POSTER 15-52

\section{Is Esophageal Stethoscopy Effective in the In-flight Setting?}

*Alan Stimson, MD, Stephen H. Thomas, MD, C. Keith Stone, MD, Dolly Bryan-Bergen, CFRN, Hershell Cassell, MD, Richard Hunt, MD, Susan Brinkley, $R N$

Division of Air Medical Services, Department of Emergency Medicine, East Carolina University School of Medicine, University Medical Center of Eastern Carolina, Greenville, North Carolina

Purpose: Previous research has confirmed the inability of flight nurses in an airborne BO-105 helicopter to hear breath sounds using normal or amplified transthoracic stethoscopy. The purpose of this study was to determine if esophageal stethoscopy enabled effective auscultation of breath sounds in an artificial, in-flight environment.

Methods: The cabin sound environment of an in-flight BO-105 helicopter was recorded and recreated in an audiology laboratory, where five flight nurses were evaluated listening to taped breath sounds via an esophageal stethoscope. This audio-tape model, validated in a previously published study, used a tape consisting of 24,20 second segments. The beginning of each segment was marked with a beep signal. Each segment consisted of 20 seconds of silence or breath sounds. The distal (esophageal dwelling) end of the esophageal stethoscope was attached to the tape recorder: the decibel level of breath sounds heard at the stethoscope ear piece was calibrated to equate to the sound level of actual esophageal breath sounds recorded on a volunteer.

Results: All nurses correctly identified the 24 taped segments as silent or including breath sounds $100 \%$ of the time.

Conclusion: In the artificial environment tested, esophageal stethoscopy enabled $100 \%$ accuracy in identification of breath sounds, as compared with previously reported $0 \%$ efficacy for standard transthoracic auscultation. Study in the actual patient care environment is indicated to confirm the usefulness of esophageal stethoscopy in the in-flight setting.
POSTER 16-25

\section{Paramedic Oral Endotracheal Intubation Rates in an EMS Quality Improvement Program}

\author{
*Matt C. Gratton, MD, Jack P. Campbell, MD, 1,2 \\ Dan Lindholm, NREMT-P, ${ }^{2}$ William A. Watson, \\ Pharm $D^{1}$ \\ 1. University of Missouri-Kansas City School of Medicine/ \\ Truman Medical Center, Missouri \\ 2. Kansas City, Missouri, Department of Health, EMS Division
}

Background: Monitoring system and individual paramedic oral endotracheal intubation (OETI) success rates has been suggested to evaluate the quality of prehospital care. Variation in individual OETI rates may be due to normal system variation or special cause variation.

Objective: To determine individual OETI rates, to identify outliers, and to determine if outliers are due to special cause variation.

Methods: Retrospective review of all attempted OETI in the Kansas City, Missouri EMS system from 1 January 1992 through 31 December 1992. Successful intubation was defined as ET tube in the trachea on hospital arrival. Frequency and $95 \%$ confidence intervals are presented. Median and interquartile range (IQR) are presented on non-normally distributed data. The Chi-square test was used.

Setting: Urban, all advanced life support (ALS), public utility model EMS system with fire department, first responders serving a city of 450,000 .

Results: The 98 paramedics were successful in 559 of 627 OETI patients $(89.1 \%$; CI $=86.7-91.5 \%)$. Success rates were significantly better for nontraumatic cardiac arrest (93 medics 439/468, 93.8\%; CI $=91.6-96.0 \%$ ) than for other medical patients (35 medics: $38 / 54$, $70.4 \%, \mathrm{CI}=58.2-82.6 \% ; p<0.0005)$; pediatric patients (18 medics $20 / 25,80.0 \%, \mathrm{CI}=64.3-95.7 \% ; p=.025$ ); or trauma patients ( 46 medics $62 / 80,77.5 \%$; $\mathrm{CI}=$ $68.3-86.7 \% ; p<0.0005)$. Individual medic success rates were compared for medics with $\geq 5$ patients. Medic success rates were non-normally distributed. For 57 medics with $\geq 5$ OETI patients, median success rate was $100 \%$ (IQR $80-100 \%$ ). Two paramedics were below the 2.5 percentile, both with one or more trauma or nonarrested medical patients. Forty-seven medics with $\geq 5$ OETI non-traumatic cardiac-arrest patients had a median success rate of $100 \%$ (IQR $87-100 \%$ ). Three medics were $<2.5$ percentile.

Conclusion: In an active EMS system, $42 \%$ of paramedics could not be compared due to a small number of patients. Special causes may be the reason for some low outliers (i.e., more difficult intubation circumstances). Medical directors should be very careful when using individual success rates to evaluate paramedics. 